\title{
Valorisation of Virgin Coconut Oil Application in Mayonnaise Production as Functional Ingredient
}

\author{
Belal J. Muhialdin ${ }^{1}$, Ley Li Ying ${ }^{2}$, Abd-Elaziem Farouk ${ }^{3}$, Anis Shobirin Meor Hussin ${ }^{1, *}$ \\ ${ }^{1}$ Faculty of Food Science and Technology, Universiti Putra Malaysia, Selangor, Malaysia \\ ${ }^{2}$ Molecular Biotechnology Research Unit, Faculty of Science, Taif University, Alhawiya, Kingdom of Saudi Arabia \\ ${ }^{3}$ Halal Products Research Institute, Universiti Putra Malaysia, 43400 UPM Serdang, Malaysia \\ *Corresponding author: shobirin@upm.edu.my
}

Received December 03, 2018; Revised January 06, 2019; Accepted January 25, 2019

\begin{abstract}
Mayonnaise is favourable by large number of consumers due to the pleasant taste that can enhance the appetite. However, several commercial mayonnaises are produced by using high concentrations of oil that may cause high threats of developing several diseases due to the link to health issues. On the other hand, oils known for health promoting properties such as virgin coconut oil are underutilized even though having high potential as plant-based alternative healthy oil that can be used in mayonnaise production. In this study, virgin coconut oil was used to partially and/or fully replace soybean oil that is commonly used in mayonnaise production. Three mayonnaise samples were prepared by using three oil combinations including 100\% virgin coconut oil, 50\%:50\% soybean oil:virgin coconut oil and $100 \%$ soybean oil. The antioxidant activity, physiochemical properties, lipid profile and sensory evaluation of the mayonnaise samples were determined by using standard methods. The results showed significantly higher antioxidant activity for the mayonnaise sample prepared with $100 \%$ virgin coconut oil in comparison to other two samples. No significant differences were observed for the physical properties including $\mathrm{pH}$ and colour. However, $100 \%$ virgin coconut oil mayonnaise sample had the largest droplet size, lowest texture firmness and lowest viscosity among the samples. The sensory evaluation demonstrated higher preference to the $100 \%$ soybean oil mayonnaise that scored total of 7.050 , while the $100 \%$ virgin coconut oil mayonnaise scored 5.57 for overall acceptability. The findings of this study demonstrated high potential for using virgin coconut oil in mayonnaise production to improve the quality and the biological functions of the product while maintaining the organoleptic properties.
\end{abstract}

Keywords: mayonnaise, functional foods, bioactive ingredients, coconut oil, healthy foods

Cite This Article: Belal J. Muhialdin, Ley Li Ying, Abd-Elaziem Farouk, and Anis Shobirin Meor Hussin, "Valorisation of Virgin Coconut Oil Application in Mayonnaise Production as Functional Ingredient." Journal of Food and Nutrition Research, vol. 7, no. 1 (2019): 65-70. doi: 10.12691/jfnr-7-1-8.

\section{Introduction}

Mayonnaise is a sauce with pleasant taste commonly used as salads dressing and also for sandwiches preparation. It is creamy semi-solid product and is made by mixing vegetable oil, egg yolk, vinegar and salt [1]. Mayonnaise is very popular among people all around the world while the demand for mayonnaise by Malaysian consumers is recently increased due to the introduction of international food restaurants including fast food, Japanese and Middle East restaurants. The main ingredients in mayonnaise are vegetable oils, water, emulsifier (egg yolk), thickeners (gum and starch), vinegar, salt and sugar, and flavouring agents. Vegetable oils such as soybean oil canola oil, cottonseed oil and sunflower oil are the main constituent for mayonnaise production that could be approximately up to $75 \%$ [2]. The oil plays very important roles in mayonnaise characteristics such as viscosity, texture, lubricity, appearance, flavour and shelf life [3]. Therefore, oil is used in very high quantities in the mayonnaise production to ensure the quality and stability of the product. However, the regular consumption of mayonnaise can be source of health problems because foods rich in fats are related to number of diseases such as cardiovascular diseases.

On the other hand, Virgin Coconut Oil (VCO) is well known for the unique characteristics and demonstrated several health benefits confirmed by in vitro and in vivo studies [4]. Shilling et al., [5] reported the antibacterial activity of VCO against Clostridium difficile and the active compounds were lauric acid, capric acid and caprylic acid. In another study, the functional properties of VCO including anti-inflammatory, analgesic, and antipyretic properties were determined in rat study [6]. The demand for VCO is growing due to the potential applications in functional food such as bakery, dairy, fats and oils, and sauces [7]. The antimicrobial activity and the antioxidant capacity of VCO can add value to the food products and it has high potential to extend the shelf life of certain food products. Modern consumers are increasing awareness about healthy foods and that has led to high demand for 
foods with known health benefits. VCO has the potential to be used in mayonnaise production as functional ingredient and for the best of our knowledge there are very limited data about the application of VCO in mayonnaise production. Great number of research have been carried out to find plant-based alternatives for the common oils used in mayonnaise production, but in the best of the author knowledge this is the first study to determine the potential use of VCO. Therefore, Mayonnaise samples were produced by using standardized recipe but with three different oil formulations were selected including 100\% soybean oil, $100 \%$ virgin coconut oil and 50\%:50\% soybean oil:virgin coconut oil. The aim of this work was to study the physiochemical properties of mayonnaise made with virgin coconut oil. The antioxidant activity, proximate analysis, physiochemical properties and sensory evaluation of the mayonnaise were further determined.

\section{Materials and Methods}

\subsection{Materials and Recipe}

The materials including the Soybean Oil (SO), Virgin Coconut Oil (VCO), egg yolk, salt, mustard, vinegar, sugar and white pepper were purchased from a local bakery shop located in the state of Selangor on 25 August 2016. A total of $500 \mathrm{~g}$ of each mayonnaise sample was prepared in this study following the method described by Akhtar et al., [8], with slight modifications. The formula of the three samples was done using same ingredients percentage and the main difference was the percentage of oils used for each sample (Table 1).

Table 1. The percentage of the ingredients used for the production of the mayonnaise samples

\begin{tabular}{lccc}
\hline Ingredients & \multicolumn{3}{c}{ Percentage (\%) } \\
\hline & Sample 1 & Sample 2 & Sample 3 \\
& $100 \%$ SBO & $\begin{array}{c}50 \%: 50 \% \\
\text { SBO:VCO }\end{array}$ & $100 \%$ VCO \\
\cline { 2 - 4 } Virgin coconut oil & 0.0 & 35.0 & 70.0 \\
Soybean oil & 70.0 & 35.0 & 0.0 \\
Egg yolk & 10.5 & 10.5 & 10.5 \\
Salt & 2.0 & 2.0 & 2.0 \\
Sugar & 1.5 & 1.5 & 1.5 \\
Mustard powder & 1.5 & 1.5 & 1.5 \\
Distilled water & 3.5 & 3.5 & 3.5 \\
Vinegar & 11.0 & 11.0 & 11.0 \\
\hline
\end{tabular}

\subsection{Mayonnaise Preparation}

The mayonnaise was prepared by following the recipe described in previous study [8]. The ingredients including egg yolk, salt, sugar, mustard powder and distilled water were mixed and placed kitchen blender, and then $10 \%$ of oil was added gradually and blended at medium speed, and another $50 \%$ of oil was added and further blended for 3 minutes at medium speed. The remaining oil (10\%) was added to the mixture and blended at high speed for 4 minutes, while the vinegar was the last ingredient to be added into the mixture. The method of adding the ingredients in this sequence is suggested to improve the emulsion stability of mayonnaise [9]. A total of $500 \mathrm{~g}$ of each mayonnaise samples were prepared in triplicate and transferred into sterilised plastic containers with caps and stored at room temperature until analysis. The $\mathrm{pH}$ value of each mayonnaise sample was measured using $\mathrm{pH}$ meter (3505 pH meter, Jenway, UK).

\subsection{Antioxidant Activity}

The antioxidant activity was evaluated for the three mayonnaise samples using three methods including DPPH, ABTS and Total Phenolic Compounds (TPC). The samples $(50 \mathrm{mg})$ were diluted in $1 \mathrm{~mL}$ ethanol and placed in 96 wells micro-titter plates. Water served as negative control and ethanol was the positive control. The radical scavenging activity was determined by using the DPPH method described in previous study [10]. The antioxidant activity of mayonnaise samples was further confirmed by using ABTS method. The TPC was determined by using Folin-Ciocalteu reagent following the method described by Marina et al., [10].

\subsection{Determination of Iodine Value}

The iodine value was measured for the three oil samples namely 100\% SBO, 50\%:50\% SBO:VCO and $100 \%$ VCO to determine the amount of unsaturated fatty acids by following AOAC standard method [11]. Briefly, $0.1 \mathrm{~g}$ of the sample was dissolved in $20 \mathrm{~mL}$ cyclohaxane and $25 \mathrm{~mL}$ of Wij's solution was added into the sample, and kept in the dark for $30 \mathrm{~min}$. A total of $20 \mathrm{~mL}$ potassium iodide solution was added to the mixture, and followed by adding $100 \mathrm{~mL}$ of distilled water. The liberated iodine was titrated with $0.25 \mathrm{M}$ sodium thiosulphate solution to a pale yellow colour. A total of 3 drops of starch solution added and the titration is continued until the solution turn colourless. The blank sample was prepared without adding the sample, and iodine value was calculated based on equation as shown below:

$$
\mathrm{IV}=(\mathrm{B}-\mathrm{T}) \times 100 / \mathrm{W}
$$

Where $\mathrm{B}=$ blank titrate of $0.25 \mathrm{M}$ sodium thiosulphate, $\mathrm{T}=$ sample titrate of $0.25 \mathrm{M}$ sodium thiosulphate, and $\mathrm{W}=$ weight in grams of sample of oil.

\subsection{Determination of Peroxide Value}

The peroxide value of the three oil samples was determined to evaluate the formation of rancidity and the effects on the mayonnaise stability [11]. A total of $5 \mathrm{~g}$ of the sample was dissolved in $30 \mathrm{~mL} 0.1 \mathrm{M}$ acetic acid-chloroform solution in conical flask and $0.5 \mathrm{ml}$ of potassium iodide solution was added. This was followed by adding of $30 \mathrm{~mL}$ of distilled water immediately, and 3 drops of $10 \%$ starch solution added. The liberated iodine was titrated with $0.1 \mathrm{M}$ sodium thiosulphate solution until the solution turn colourless. The blank sample was prepared without the addition of oil sample, and peroxide value was calculated based on equation as shown below:

$(\mathrm{S}-\mathrm{B}) \times \mathrm{M}$ thiosulphate $\times 1000 / \mathrm{W}$ 
Where $\mathrm{S}=$ sample titrate of $0.1 \mathrm{M}$ sodium thipsulphate, $\mathrm{B}=$ blank titrate of $0.1 \mathrm{M}$ sodium thipsulphate, $\mathrm{M}=$ molarity of sodium thipsulphate, $\mathrm{W}=$ weight of oil sample.

\subsection{Determination of Saponification Value}

The saponification value was determined according to AOAC method [11]. In a conical flask, $5 \mathrm{~g}$ of sample was mixed with $50 \mathrm{~mL}$ of $0.5 \mathrm{M}$ alcoholic $\mathrm{KOH}$, and the flask was connected to the reflux condenser and allowed to reflux for $30 \mathrm{~min}$. The solution was cooled and titrated with $0.5 \mathrm{M} \mathrm{HCl}$ until the end-point was reached, with phenolphthalein as indicator. The saponification value was calculated based on equation below:

\section{Saponification value $=(\mathrm{S}-\mathrm{B}) \times \mathrm{M} \mathrm{HCl} \times 56.1 / \mathrm{W}$}

Where $\mathrm{S}=$ sample titrate of $0.5 \mathrm{M} \mathrm{HCl}, \mathrm{B}=$ blank titrate of $0.1 \mathrm{M}$ sodium thipsulphate, $\mathrm{M}=$ molarity of $\mathrm{HCl}$, $\mathrm{W}=$ weight of oil sample.

\subsection{Particle Size Distribution and Texture}

The particle size distribution of the mayonnaise samples was determined by a particle size analyser (Mastersizer, 2000, Worcestershire, UK) by following the method described by Liu et al. [9] with some modifications. The mayonnaise samples $(0.04 \mathrm{~g})$ were dissolved in $150 \mathrm{~mL}$ of $0.1 \%$ sodium dodecyl sulfate (SDS) buffer solution, and sample solution was dispersed in distilled water $9200 \mathrm{~mL}$ at $2500 \mathrm{rpm}$ until an obstruction rate of $5 \%$ was obtained. The Malvern software used the relative index of VCO (1.500) and the relative index of dispersant water (1.330) to calculate the dispersion span. Particle size measurement was reported as the volume-weighted mean diameter: $\mathrm{d}_{4,3}=\sum \mathrm{n}_{\mathrm{i}} \mathrm{d}_{\mathrm{i}}^{4} / \sum \mathrm{n}_{\mathrm{i}} \mathrm{d}^{3}{ }_{\mathrm{i}}$, where $\mathrm{n}_{\mathrm{i}}$ was the number of droplets of diameter $\mathrm{d}_{\mathrm{i}}$. Furthermore, the texture of the mayonnaise samples was determined by using TA.XT2 $\mathrm{i}$ Texture Analyser (Stable Micro Systems, Surrey, UK). The samples were placed in a plastic container at a depth of 75 $\mathrm{mm}$ and were tested using a $\mathrm{P} / 36 \mathrm{R}$ aluminium probe (10 $\mathrm{mm}$ penetration, $1 \mathrm{~mm} / \mathrm{s}$ crosshead speed).

\subsection{Mayonnaise Rheological Properties}

Mayonnaise rheological property was measured by using rheometer (MCR 300, Physica/Anton Paar, Ostfildern, Germany) following the method described by Liu et al., [9]. The mayonnaise samples were poured into the test viscometer cup, and all samples were allowed to rest for 5 min before starting the measurements to allow temperature equilibration and induced stress to relax. All tests were carried out at room temperature $\left(25^{\circ} \mathrm{C}\right)$ and in triplicate.

\subsection{Sensory Evaluation}

The sensory evaluation was carried out to determine the consumer decision for the mayonnaise samples that are preferred. A total of 40 untrained panellists aged 18-26 performed the sensory test to determine the appearance, colour, odour, texture, taste and overall acceptance on 9-point hedonic scale which $1=$ the least/lowest like and 9=the most/highest like. All mayonnaise samples were coded with three-digit random numbers and presented to panellists on a tray in individual booths to avoid bias. The panellists were briefed about the sensory attributes then called randomly to perform the sensory test. Water was provided between samples to cleanse the palate as described in previous study [9].

\subsection{Statistical Analysis}

The mayonnaise samples were prepared in three batches for each formula and the data was subjected to a one-way analysis of variance (ANOVA) using Minitab (Version 17, Minitab Pennsylvania, USA) statistical software. Significant differences between the mean values $(\mathrm{P}<0.05)$ were determined using Tukey's test. The $\pm \mathrm{SD}$ symbol represented the standard deviations of the mean values obtained from the triplicate samples.

\section{Results and Discussion}

In modern days, social media helped to enhance the public knowledge regarding healthy foods and led the consumers to demand for foods that have health benefits beyond the common nutrients. On the other hand, the food manufacturers are demanding for bioactive ingredients for the applications in several value-added functional foods. In this study mayonnaise was produced by partially or fully replacing Soybean Oil (SBO) with Virgin Coconut Oil (VCO) that is well-known for the high content of phenolic compounds and the strong antioxidant activity. Total Phenolic Compounds (TPC) content for the three mayonnaise samples was $31.52 \mathrm{mg}$ GAE/100g oil, $46.99 \pm 1.54 \mathrm{mg}$ GAE $/ 100 \mathrm{~g}$ oil and $50.74 \pm 1.54 \mathrm{mg}$ GAE $/ 100 \mathrm{~g}$ for the $100 \%$ SBO mayonnaise, 50\%:50\% VCO:SBO mayonnaise and $100 \%$ VCO mayonnaise, respectively (Table 2). There was a significant difference $(p<0.005)$ for the TPC content between the $100 \%$ SBO mayonnaise and the $100 \% \mathrm{VCO}$ mayonnaise due to the high phenolic content in VCO. TPC content is very important for functional food ingredients because they have strong correlation with the antioxidant activity as demonstrated in great number of studies [7]. The results of this study were in agreement with previous studies and TPC content demonstrated strong impact on the antioxidant activity of $100 \%$ VCO mayonnaise sample.

The DPPH radical scavenging activity was carried out to evaluate the ability of three mayonnaise samples to act as free radical scavengers or hydrogen donors which is expressed as antioxidant activity. The DPPH results showed slightly higher antioxidant activity for $100 \% \mathrm{VCO}$ followed by 50\%:50\% SBO:VCO mayonnaise and the lowest activity was observed for the $100 \%$ SBO mayonnaise sample (Table 2). The antioxidant activity of the mayonnaise samples is possibly due to the use of different oils in the preparation of each sample. Marina et al., [10] reported high antioxidant activity of the VCO in comparison to refined, bleached and deodorized coconut oils. The author observed that very high correlations between total phenolic content and scavenging activity and reducing power. The antioxidant activity was further confirmed by $\mathrm{ABTS}^{+}$free radical scavenging activity assay. The $100 \%$ VCO mayonnaise demonstrated the highest scavenging activity $(67.95 \pm 1.47 \%)$, followed by 
50\%:50\% SBO:VCO mayonnaise $(65.28 \pm 1.47 \%)$ and the $100 \%$ SBO mayonnaise $(56.91 \pm 1.43 \%)$. In previous study, VCO diet significantly enhanced the antioxidant status of rats after 45 days of feeding, and the VCO demonstrated antioxidant activity in vitro and in vivo [12]. Nevertheless, the results of this study were in the same trend of previous studies about the antioxidant activity of VCO. The preparation of mayonnaise does not involve aggressive steps such as heating and therefore it will not cause bad impact on the antioxidant activity which promotes mayonnaise as good carrier for the functional ingredients. The $\mathrm{pH}$ value of the mayonnaise samples was determined because it is very crucial for the mayonnaise shelf life and structure. The $\mathrm{pH}$ of mayonnaise is preferred to be in acidic conditions to prevent the growth of spoilage microorganisms [1]. The results showed no significant effects for the oil type on the mayonnaise $\mathrm{pH}$ for three samples (Table 2).

The use of virgin coconut oil as alternative to the soybean oil in mayonnaise production may have effects on the rheological property, textural and the stability during storage (shelf life). Therefore, the lipid analysis including iodine value, peroxide value and saponification value was determined to evaluate the effects of the oils on the mayonnaise characteristics and the stability during storage. The iodine value measured the degree of unsaturation in lipids. The higher iodine value refers to high degree of unsaturation in lipids while low iodine value indicates low degree of unsaturation in lipids [13]. The results demonstrated significant differences among three mayonnaise samples (Table 3). The iodine value for the $100 \% \mathrm{VCO}$, 50\%:50\% SBO:VCO, and $100 \%$ SBO was $6.81 \pm 0.23$, $75.72 \pm 4.12$ and $140.59 \pm 2.79$, respectively. According to Malaysia standards for virgin coconut oil, the iodine value is in the range of 5.5-10.6 while the iodine value of $100 \%$ SBO is in the range of 137-143 [14]. The results are in agreement with previous data and the iodine value of VCO significantly low in comparison to $100 \%$ SBO. The low iodine value of VCO is due to the nature of the fatty acid content that are mostly saturated fatty acid, while $100 \%$ SBO naturally contained high amount of unsaturated fatty acid.

The Peroxide Value (PV) was studied to measure the primary oxidative degradation of the oil samples used in the production of the mayonnaise samples [15]. The peroxide value results showed no significant $(\mathrm{p}>0.05)$ difference among the three oil samples. The PV has correlation to the oxidation and the stability of the oils whereas the low values indicate to high stability [16]. In this study, the PV was observed to be low for SBO and VCO that indicated their high stability. The mayonnaise production did not involve heat treatment, and it did not have effects on the peroxide value. On the other hand, Saponification Value (SP) was evaluated to determine the quality and properties of the prepared mayonnaise using different oils. Such value was expressed by the number of $\mathrm{mg}$ of potassium hydroxide which required to saponify $1 \mathrm{~g}$ of fat or oil. SP measures the length of fatty acid chains, and the lower SP value indicates longer fatty acid chains [17]. The saponification value of VCO and 50\%:50\% SBO:VCO was significantly $(\mathrm{p}<0.05)$ higher than $100 \%$ SBO. VCO demonstrated high SP due to the presence of medium chain fatty acid (12C) and the SV was in the range of $250-260 \mathrm{mg} / \mathrm{g}$ [10], while the fatty acid present in $100 \%$ SBO are of long chain (18C) and the $\mathrm{SV}$ ranged between 189 and 195mg/g [18]. The lipid profile in this study showed significant effects of the oils used for the mayonnaise production and therefore the physical properties of the mayonnaise samples were further analysed.

The physical properties of mayonnaise including rheology, emulsion stability and particle size was carried out to ensure the quality of the product. The replacement of the soybean oil with the virgin coconut oil in mayonnaise production can cause significant changes in the sensory and physical properties that may be not preferred by consumers [19]. Therefore, the physical properties were determined to evaluate the effects of different oils on the mayonnaise samples. The droplet size of the mayonnaise samples was evaluated due to the influence on the appearance, texture, and flavour profile of the product [20]. The variance of the mean diameter of droplet particle size for different mayonnaise samples were expressed by the $D_{4,3}$ value $(\mu \mathrm{m})$. The results exhibited significant differences $(\mathrm{p}<0.05)$ in droplet size for the three studied mayonnaise samples. The $100 \%$ SBO mayonnaise had the smallest droplet particle size $(6.65 \mu \mathrm{m})$, and $100 \%$ VCO mayonnaise had the largest droplet particle size $(16.24 \mu \mathrm{m})$. Di Mattia et al., [21] reported the correlation between the high droplet size and high phenolic content of oils. The results in this study were in agreement with the previous study and the high phenolic content of VCO was observed to be correlated with the large droplet size. On the other hand, texture analysis of the mayonnaise samples showed opposite corresponding to the phenolic content [21]. The results demonstrated significant $(\mathrm{p}<0.05)$ decrease in the texture firmness value of $100 \%$ VCO mayonnaise sample in comparison to $100 \%$ SBO mayonnaise sample. Moreover, the texture hardness was slightly dropped for $100 \% \mathrm{VCO}$ mayonnaise samples. The substitution of SBO by VCO in mayonnaise production showed decline for the texture firmness and hardness but all the results were in acceptable ranges. Further investigation is required for to determine the effects of different VCO that have different phenolic contents on the texture of mayonnaise. In this study, TPC content was lightly different for VCO and SBO but it demonstrated significant differences for the physical properties of the prepared mayonnaise samples.

The results of the dynamic viscosity demonstrated slightly lower viscosity in the $100 \%$ VCO mayonnaise sample and 50\%:50\% SBO:VCO mayonnaise sample in comparison to the $100 \%$ SBO mayonnaise sample (Table 4). The high viscosity is essential for mayonnaise to prevent the adhesion of the oil droplets and enhance the stability of the product [22]. The result indicated that the substitution of SBO with VCO did not have significant effects on the rheological properties of the mayonnaise samples. However, the viscosity of the three samples was low in this study because the samples were stored and analysed at room temperature. Ghoush et al., [23] reported the high viscosity of mayonnaise samples when determined at low temperatures while the high temperatures reduced the viscosity. The physical analysis showed good rheological properties for the mayonnaise prepared by using 100\% VCO. In addition, mayonnaise rheology can 
be improved by optimizing several factors including homogenization speeds, oil concentration and oil fatty acid composition. Increased homogenization speeds can improve mayonnaise gel appearance and reduce spread-ability [25]. Furthermore, $\mathrm{pH}$ value can enhance the emulsion stability when it is adjusted to point close to the isoelectric point of the egg yolk proteins. Type of oils and their chemical composition have significant effect on the rheology of the mayonnaise. In this study, the VCO demonstrated good characteristics and it was suitable for the mayonnaise production.

\subsection{Sensory Evaluation}

The success of the healthy foods in the marketplace is heavily depends on the consumer decisions that is influenced by their preference to the food characteristics such as aroma, appearance and flavour. Sensory evaluation analysis was carried out to determine the effects of replacing the common Soybean oil (SBO) used for mayonnaise production by Virgin Coconut Oil (VCO) on the preference of the consumers. $100 \%$ SBO mayonnaise achieved the highest score for the overall acceptability including appearance, colour, aroma, texture, and flavour (Table 5). The sensory evaluation for $100 \% \mathrm{VCO}$ mayonnaise showed high scores for colour and flavour, while the aroma, texture and appearance had low scores. The substitution of oil in this study demonstrated significant decline for the acceptability of the mayonnaise as determined by 40 untrained panels. According to panel testimony, the low scores were given to $100 \% \mathrm{VCO}$ mayonnaise due to the strong aroma of coconut oil while the panel was not able to distinguish the differences for the colour. The removal and/or the replacement of mayonnaise main ingredients will cause significant changes to the organoleptic properties and the preference of the consumers [19]. Therefore, the replacement of soybean oil commonly used in the mayonnaise by virgin coconut oil showed significant results indicated by the preference by the panellist. In previous study, mayonnaise prepared by using virgin olive oil also received low scores due to the bitter and spicy test observed by the panellist. The low scores for the aroma and flavour of the mayonnaise samples using alternative oils might be related to the content and the type of the phenolic compounds which present in the oil. Therefore, it can have effects on the sensory response [26]. The sensory analysis results showed significant correlations between the texture of the mayonnaise and the rheological properties of the oil and that was in agreement with previous studies [9].

Table 2. The antioxidant activity of mayonnaise determined by three methods including TPC, DPPH and ABTS

\begin{tabular}{llll}
\hline Samples & TPC (mgGAE/100g oil) & DPPH (\%) & ABTS (\%) \\
\hline $100 \%$ SBO & $31.52^{\mathrm{b}} \pm 2.39$ & $50.49^{\mathrm{a}} \pm 2.20$ & $56.91^{\mathrm{b}} \pm 1.43$ \\
$50 \%: 50 \%$ SBO:VCO & $46.99^{\mathrm{a}} \pm 1.54$ & $55.35^{\mathrm{a}} \pm 1.80$ & $65.28^{\mathrm{a}} \pm 1.47$ \\
$100 \%$ VCO & $50.75^{\mathrm{a}} \pm 1.54$ & $58.69^{\mathrm{a}} \pm 3.09$ & $67.95^{\mathrm{a}} \pm 1.47$ \\
\hline
\end{tabular}

Note: Each value is the mean of triplicate determinations ${ }^{ \pm}$standard deviation.

The same superscripts in the same column denote no significant different $(\mathrm{p}>0.05)$.

Table 3. The iodine value, peroxide value and saponification value of the three mayonnaise samples

\begin{tabular}{llll}
\hline Samples & Iodine Value & Peroxide Value (meq/g) & Saponification value (mg/g) \\
\hline $100 \%$ SBO & $140.59^{\mathrm{a}} \pm 2.79$ & $1.12^{\mathrm{a}} \pm 0.1190$ & $194.27^{\mathrm{c}} \pm 2.75$ \\
$50 \%: 50 \%$ SBO:VCO & $75.72^{\mathrm{b}} \pm 4.12$ & $1.04^{\mathrm{a}} \pm 0.09$ & $242.73^{\mathrm{b}} \pm 0.07$ \\
$100 \%$ VCO & $6.81^{\mathrm{c}} \pm 0.23$ & $1.13^{\mathrm{a}} \pm 0.11$ & $254.19^{\mathrm{a}} \pm 6.07$ \\
\hline
\end{tabular}

Note: Each value is the mean of triplicate determinations ${ }^{ \pm}$standard deviation.

The same superscripts in the same column denote no significant different $(\mathrm{p}>0.05)$.

Table 4. Physiochemical properties of different mayonnaise samples

\begin{tabular}{|c|c|c|c|}
\hline \multirow[t]{2}{*}{ Physiochemical properties } & \multicolumn{3}{|c|}{ Mayonnaise samples } \\
\hline & $100 \%$ SBO & $50 \%: 50 \%$ SBO:VCO & $100 \% \mathrm{VCO}$ \\
\hline Droplet size $(\mu \mathrm{m})$ & $6.65^{\mathrm{a}} \pm 0.11$ & $8.79^{\mathrm{b}} \pm 0.12$ & $16.24^{\mathrm{c}} \pm 0.05$ \\
\hline Texture firmness (g) & $132.77^{\mathrm{a}} \pm 5.15$ & $116.59^{\mathrm{b}} \pm 4.05$ & $101.72^{\mathrm{c}} \pm 1.82$ \\
\hline Texture hardness $(\mathrm{g} / \mathrm{s})$ & $873.20^{\mathrm{a}} \pm 33.20$ & $832.60^{\mathrm{ab}} \pm 17.8$ & $811.43^{b} \pm 12.71$ \\
\hline Viscosity (mPas) & $87.27^{\mathrm{a}} \pm 0.78$ & $85.36^{\mathrm{b}} \pm 0.23$ & $83.50^{c} \pm 0.52$ \\
\hline
\end{tabular}

Note: Each value is the mean of triplicate determinations ${ }^{ \pm}$standard deviation.

The same superscripts in the same column denote no significant different $(p>0.05)$.

Assays were performed in triplicate. Mean GSD values followed by the same letter in each column are not significant different at $P>0.05$ by ANOVA and Tukey's test.

Table 5. Sensory evaluation of mayonnaise samples

\begin{tabular}{lccc}
\hline & & Mayonnaise samples \\
\hline & $\mathbf{1 0 0 \%}$ SBO & $\mathbf{5 0 \% : 5 0 \% ~ S B O : V C O}$ & $\mathbf{1 0 0 \%}$ VCO \\
\cline { 2 - 5 } Appearance & $6.975 \pm 1.63^{\mathrm{b}}$ & $6.03 \pm 1.47^{\mathrm{a}}$ & $6.09 \pm 1.19^{\mathrm{a}}$ \\
Croma & $7.50 \pm 1.35^{\mathrm{a}}$ & $5.80 \pm 1.82^{\mathrm{a}}$ & $7.10 \pm 1.44^{\mathrm{a}}$ \\
Telour & $7.55 \pm 1.61^{\mathrm{b}}$ & $6.47 \pm 1.22^{\mathrm{a}}$ & $7.20 \pm 1.27^{\mathrm{a}}$ \\
Flavour & $6.973 \pm 1.64^{\mathrm{b}}$ & $5.85 \pm 2.19^{\mathrm{a}}$ & $6.20 \pm 1.30^{\mathrm{a}}$ \\
Overall Acceptability & $7.05 \pm 1.54^{\mathrm{a}}$ & $5.57 \pm 1.66^{\mathrm{ab}}$ & $5.8 \pm 1.94^{\mathrm{a}}$ \\
\hline
\end{tabular}


The preparation of mayonnaise by using virgin coconut oil showed lower firmness and consistency in comparison to soybean oil. The mayonnaise prepared by using Virgin Coconut Oil (VCO) demonstrated high TPC content and high antioxidant activity in comparison to the mayonnaise prepared by using Soybean Oil (SBO). The lipid profile, physical properties and technological functionality of $\mathrm{VCO}$ were in the acceptable range for the mayonnaise production. However, $100 \%$ SBO mayonnaise was slightly preferred in comparison to $100 \% \mathrm{VCO}$ due to strong flavour and aroma of VCO. The findings of this study suggest the improvement of the mayonnaise biological activity by using VCO as the main ingredient. Moreover, 100\% VCO mayonnaise has the potential for health enhancement due to high antioxidant activity. The findings of this study highly recommend the application of VCO to produce novel mayonnaise with value-added properties. Further study is required to improve the $100 \%$ VCO mayonnaise formulation with considerations to enhance the consumer preference.

\section{Acknowledgements}

The authors would like to thank the Faculty of Food Science and Technology, Universiti Putra Malaysia (UPM) for their support during the laboratory work

\section{Statement of Competing Interests}

The authors have no competing interest to report.

\section{References}

[1] Depree, J. A., and Savage, G. P. Physical and flavour stability of mayonnaise. Trends in Food Science \& Technology, 12(5-6), 157163. May. 2001.

[2] Smittle, R. B. Microbiological safety of mayonnaise, salad dressings, and sauces produced in the United States: a review. Journal of food protection, 63(8), 1144-1153. August. 2002.

[3] McClements, D. J., and Demetriades, K. An integrated approach to the development of reduced-fat food emulsions. Critical Reviews in Food Science and Nutrition, 38(6), 511-536. August. 1998.

[4] Carandang, E. V. Health benefits of virgin coconut oil. INDIAN COCONUT JOURNAL-COCHIN-, 38(9), 8. January. 2008.

[5] Shilling, M., Matt, L., Rubin, E., Visitacion, M. P., Haller, N. A., Grey, S. F., and Woolverton, C. J. Antimicrobial effects of virgin coconut oil and its medium-chain fatty acids on Clostridium difficile. Journal of medicinal food, 16(12), 1079-1085. December. 2013.

[6] Intahphuak, S., Khonsung, P., and Panthong, A. Anti-inflammatory, analgesic, and antipyretic activities of virgin coconut oil. Pharmaceutical biology, 48(2), 151-157. February. 2010.

[7] Marina, A. M., Man, Y. C., and Amin, I. Virgin coconut oil: emerging functional food oil. Trends in Food Science \& Technology, 20(10), 481-487. October. 2009.

[8] Akhtar, M., Murray, B. S., and Dowu, S. A novel continuous process for making mayonnaise and salad cream using the spinning disc reactor: Effect of heat treatment.
Food Hydrocolloids, 42, 223-228. December. 2014.

[9] Liu, H., Xu, X. M., and Guo, S. D. Rheological, texture and sensory properties of low-fat mayonnaise with different fat mimetics. LWT-Food Science and Technology, 40(6), 946-954. August. 2007.

[10] Marina, A. M., Che Man, Y. B., Nazimah, S. A. H., and Amin, I. Antioxidant capacity and phenolic acids of virgin coconut oil. International Journal of Food Sciences and Nutrition, 60(sup2), 114-123. January. 2009.

[11] Chemists, A. A., \& Horwitz, W. Official methods of analysis. Vol. I. 15th ed. AOAC, Arlington, VA. 1990.

[12] Nevin, K. G., and Rajamohan, T. Virgin coconut oil supplemented diet increases the antioxidant status in rats. Food chemistry, 99(2), 260-266. January. 2006.

[13] Ryan, T. W., Dodge, L. G., and Callahan, T. J. The effects of vegetable oil properties on injection and combustion in two different diesel engines. Journal of the American Oil Chemists Society, 61(10), 1610-1619. October. 1984

[14] Amri, I. N. The lauric (coconut and palm kernel) oils. Vegetable oils in food technology. West Sussex: Wiley-Blackwell, 169-97. February. 2011.

[15] Matthäus, B. Oxidation of edible oils. Oxidation in foods and beverages and antioxidant applications. Volume 2: Management in different industry sectors, 183-238. 2010.

[16] Popa, M., Glevitzky, I., Dumitrel, G. A., Glevitzky, M., and Popa, D. STUDY ON PEROXIDE VALUES FOR DIFFERENT OILS AND FACTORS AFFECTING THE QUALITY OF SUNFLOWER OIL. SCIENTIFIC PAPERS-SERIES E-LAND RECLAMATION EARTH OBSERVATION \& SURVEYING ENVIRONMENTAL ENGINEERING, 6, 137-140. January. 2017.

[17] Belitz, H. D., Grosch, W., and Schieberle, P. Edible fats and oils. Food Chemistry, 640-669. 2009.

[18] Ezeokeke, C. T., and Onuoha, I. E. Oil Extraction and Biochemical Analysis of Soybean Seed to Ascertain Its Nutritional Importance. International Journal of Agriculture and Rural Development, 19: 2427-2432. 2016

[19] Ma, Z., and Boye, J. I. Advances in the design and production of reduced-fat and reduced-cholesterol salad dressing and mayonnaise: a review. Food and Bioprocess Technology, 6(3), 648-670. March. 2013.

[20] Ng, S. P., Lai, O. M., Abas, F., Lim, H. K., and Tan, C. P. Stability of a concentrated oil-in-water emulsion model prepared using palm olein-based diacylglycerol/virgin coconut oil blends: Effects of the rheological properties, droplet size distribution and microstructure. Food research international, 64, 919-930. October. 2014.

[21] Di Mattia, C., Balestra, F., Sacchetti, G., Neri, L., Mastrocola, D., and Pittia, P. Physical and structural properties of extra-virgin olive oil based mayonnaise. LWT-Food Science and Technology, 62(1), 764-770. June. 2015.

[22] Wendin, K., Ellekjær, M. R., and Solheim, R. Fat content and homogenization effects on flavour and texture of mayonnaise with added aroma. LWT-Food Science and Technology, 32(6), 377-383. September. 1999.

[23] Ghoush, M. A., Samhouri, M., Al-Holy, M., and Herald, T. Formulation and fuzzy modeling of emulsion stability and viscosity of a gum-protein emulsifier in a model mayonnaise system. Journal of Food Engineering, 84(2), 348-357. January. 2008

[24] Shinn, S. E., Proctor, A., Gilley, A. D., Cho, S., Martin, E., and Anthony, N. B. Effect of feeding CLA on plasma and granules fatty acid composition of eggs and prepared mayonnaise quality. Food chemistry, 197, 57-65. April. 2016.

[25] Depree, J. A., and Savage, G.P. Physical and flavour stability of mayonnaise. Trends in Food Science \& Technology, 12, 157-163. May. 2001.

[26] Chambers, E., and Koppel, K. Associations of volatile compounds with sensory aroma and flavor: The complex nature of flavor. Molecules, 18, 4887-905. May. 2013. 